\title{
On the Drifting Parts in the Spatial Power Spectrum of Geomagnetic Secular Variation
}

\author{
Heikki NeVANLINNA* \\ University of Helsinki, Department of Geophysics, Fabianinkatu 24A, SF-00100 Helsinki 10, Finland
}

(Received November 10, 1986; Revised March 10, 1987)

\begin{abstract}
The spatial spectrum of secular variation (SV) from 1955 to 1985 was studied using recent spherical harmonic models. The drifting part of the spectrum was decomposed into longitudinal (LD) and meridional (MD) parts. In the IGRF 1980 the mean relative magnitudes of the $\mathrm{LD}, \mathrm{MD}$ and non-drifting (ND) parts were $51 \%$, $29 \%$ and $20 \%$, respectively. During the last 20 years there has been a slight increase in the LD and ND parts. The ND part dominates $(63 \%$ in 1985) the SV of the dipole field. An interesting feature in the recent dipole $S V$ is a rapid decrease of the LD part and an increase in the MD part. In 1965 the relative magnitudes were 2\% (MD) and $11 \%$ (LD) in 1985 they were $37 \%$ and $0.2 \%$, respectively.

A correlation was found between the jerk signal in 1970 and a change in the curvature of the LD and MD curves. The ND part did not show any change around 1970.
\end{abstract}

\section{Introduction}

Recently many papers have been published in which properties of the spatial power spectra of the geomagnetic field and secular variation have been discussed (e.g. MEYer et al., 1983; MEYER, 1985, 1986; OSTROWSKI, 1982; AlLDREDGE, 1984a, b, 1985,1986 ; NEVANLINNA, 1987). The spatial spectrum $W(n)$, or the mean-square of the total field $B_{n}$ belonging to each harmonic $n$ and averaged over the globe is given by:

$$
W(n)=B_{n}^{2}=X_{n}^{2}+Y_{n}^{2}+Z_{n}^{2}=(n+1) \sum_{m=0}^{n}\left\{\left(g_{n}^{m}\right)^{2}+\left(h_{n}^{m}\right)^{2}\right\}
$$

where $g_{n}^{m}$ and $h_{n}^{m}$ are the Gaussian coefficients of a spherical harmonic expansion of the geomagnetic field (see e.g. MEYER et al., 1983 and their references).

In analogy with (1), we can define the spatial spectrum $Q(n)$ of secular variation (SV) as follows:

$$
Q(n)=\dot{B}_{n}^{2}=\dot{X}_{n}^{2}+\dot{Y}_{n}^{2}+\dot{Z}_{n}^{2}=(n+1) \sum_{m=0}^{n}\left\{\left(\dot{g}_{n}^{m}\right)^{2}+\left(h_{n}^{m}\right)^{2}\right\}
$$

*Present address: Finnish Meteorological Institute, Division of Geomagnetism, Box 503, SF-00101 Helsinki 10, Finland. 
where the dots denote the time derivative, i.e. the yearly secular variation of the field coefficients.

Equation (2) represents of course not the real time derivative of $W(n)$ which is given by:

$$
\dot{W}(n)=2 B_{n} \dot{B}_{n}=2(n+1) \sum_{m=0}^{n}\left\{g_{n}^{m} \dot{g}_{n}^{m}+h_{n}^{m} h_{n}^{m}\right\}
$$

(see MeYer et al., 1983; MEYER, 1986; AlldredGe, 1986).

As shown by NEVANLINNA (1987) the spectrum $Q(n)$ gives a more complete picture of the SV than does $\dot{W}(n)$ because $Q(n)$ contains contributions due to both standing, i.e. non-drifting (ND), and drifting (D) parts of the field. The spectrum $\dot{W}(n)$, which is also the time derivative of the magnetic energy density if multiplied by the constant $1 / 2 \mu_{0}$ ( $\mu_{0}$ being the vacuum permeability), is connected only with ND changes in the field.

In this paper the technique introduced by NEVANLINNA (1987) will be applied to decompose the secular variation spectrum $Q$ into two parts: standing and drifting ones. A new method is presented to separate the longitudinal (LD) and meridional (MD) parts in the D part. The MD and LD components will be studied using recent spherical harmonic models for the interval 1955-1985 (IAGA DIVISION I WORKING GROUP 1, 1985) as sources of the drifting field.

2. The Spatial Spectrum of SV Caused by the Drift of Harmonic Constituents

Starting from the conventional spherical harmonic representation of the geomagnetic field, we can write for the $X, Y$ and $Z$ components:

$$
\begin{aligned}
X_{n}^{m}(\theta, \lambda) & =\left[g_{n}^{m} \cos (m \lambda)+h_{n}^{m} \sin (m \lambda)\right] \mathrm{d} P_{n}^{m}(\cos \theta) / \mathrm{d} \theta \\
Y_{n}^{m}(\theta, \lambda) & =m\left[-g_{n}^{m} \sin (m \lambda)+h_{n}^{m} \cos (m \lambda)\right] P_{n}^{m}(\cos \theta) / \sin \theta \\
Z_{n}^{m}(\theta, \lambda) & =-(n+1)\left[g_{n}^{m} \cos (m \lambda)+h_{n}^{m} \sin (m \lambda)\right] P_{n}^{m}(\cos \theta)
\end{aligned}
$$

where $\theta$ is the co-latitude, $\lambda$ the east longitude and $P_{n}^{m}(\cos \theta)$ Schmidt quasinormalized associated Legendre polynomials (CHAPMAN and BARTELS, 1940).

Secular variation due to longitudinal and meridional drift with the velocities $u_{n}^{m}$ and $v_{n}^{m}$, respectively, can be expressed with the following formulae:

$$
\begin{aligned}
& \dot{X}_{n}^{m}(\theta, \lambda)=\left(\partial X_{n}^{m} / \partial \lambda\right) u_{n}^{m}+\left(\partial X_{n}^{m} / \partial \theta\right) v_{n}^{m} \\
& \dot{Y}_{n}^{m}(\theta, \lambda)=\left(\partial Y_{n}^{m} / \partial \lambda\right) u_{n}^{m}+\left(\partial Y_{n}^{m} / \partial \theta\right) v_{n}^{m} \\
& \dot{Z}_{n}^{m}(\theta, \lambda)=\left(\partial Z_{n}^{m} / \partial \lambda\right) u_{n}^{m}+\left(\partial Z_{n}^{m} / \partial \theta\right) v_{n}^{m}
\end{aligned}
$$

Then using (2) and (5), the D part of the SV spectrum is: 


$$
\begin{aligned}
Q_{\mathrm{D}}(n) & =Q_{\mathrm{LD}}(n)+Q_{\mathrm{MD}}(n) \\
& =(1 / 4 \pi) \int_{0}^{2 \pi} \int_{0}^{\pi}\left[\sum_{m=0}^{n}\left\{\left(\partial X_{n}^{m} / \partial \lambda\right)^{2}+\left(\partial Y_{n}^{m} / \partial \lambda\right)^{2}+\left(\partial Z_{n}^{m} / \partial \lambda\right)^{2}\right\}\left(u_{n}^{m}\right)^{2}\right. \\
& \left.+\left\{\left(\partial X_{n}^{m} / \partial \theta\right)^{2}+\left(\partial Y_{n}^{m} / \partial \theta\right)^{2}+\left(\partial Z_{n}^{m} / \partial \theta\right)^{2}\right\}\left(v_{n}^{m}\right)^{2}\right] \sin \theta \mathrm{d} \theta \mathrm{d} \lambda .
\end{aligned}
$$

Note that the integrals containing cross product terms like $\partial X / \partial \lambda \cdot \partial X / \partial \theta$ go to zero because of orthogonality and only those shown in (6) are left. After performing the integrations in (6) we find the following formula for the LD part of $Q_{\mathrm{D}}(n)$ :

$$
Q_{\mathrm{LD}}(n)=(n+1) \sum_{m=0}^{n} m^{2}\left(u_{n}^{m}\right)^{2}\left\{\left(g_{n}^{m}\right)^{2}+\left(h_{n}^{m}\right)^{2}\right\}
$$

which differs only by the factor $m^{2}\left(u_{n}^{m}\right)^{2}$ from the spectrum $W(n)$ given in (1) for the main field.

Unfortunately it is not possible to derive for $Q_{\mathrm{MD}}(n)$ such a simple formula like Eq. (7), but after some lengthy calculations we get for the MD part:

$$
Q_{\mathrm{MD}}(n)=\sum_{m=0}^{n}\left(v_{n}^{m}\right)^{2}[x(n, m)+y(n, m)+z(n, m)]\left\{\left(g_{n}^{m}\right)^{2}+\left(h_{n}^{m}\right)^{2}\right\}
$$

where

$$
\begin{aligned}
& x(n, m)=\left(\delta_{m-1}-1\right) n(n+1)(2 n-1) / 2(2 n+1) \\
&-(1 / 32)\left[\left(2-\delta_{m}\right)(m+2) /(m+1)^{2}+\left(2-\delta_{m-1}\right)\left\{a_{0} a_{3} /(m+1)\right.\right. \\
&\left.-a_{1} a_{4} /(m-1)-2\left(a_{1} a_{3}+n\right)\right\}^{2} /\left(m+\delta_{m}-1\right) \\
&\left.+\left(2-\delta_{m-2}\right)\left\{a_{1} a_{4}\left[a_{2} a_{5}-a_{1}\left(a_{4}+2(m+1)\right) /(m-1)\right] /(m-2)\right\}^{2}\right] \\
& y(n, m)=(1 / 8)\left(2-\delta_{m-1}\right)\left\{a_{1} a_{4}\left(m^{3}+1\right) /\left(m^{3}-1\right)+a_{0} a_{3}\right\} \\
& z(n, m)=(n+1)^{2}\{n(n+1) /(2 n+1)-m / 2\} \\
& a_{0}=n+m+1 ; \quad a_{1}=n+m ; \quad a_{2}=n+m-1 ; \\
& a_{3}=n-m ; \quad a_{4}=n-m+1 ; \quad a_{5}=n-m+2 \\
& \delta_{k}=2 \text { if } k \leq 0 ; \quad \delta_{k}=1 \text { if } k>0 .
\end{aligned}
$$

In these equations $x(n, m), y(n, m)$ and $z(n, m)$ multiplied by the other factors in (8) give the contribution of meridional drifting $X, Y$ and $Z$ components to the spectrum $Q_{\mathrm{MD}}(n)$.

From Eqs. (7) and (8) we can easily see that in the longitudinal case for a given $n$ and constant velocity, $Q_{\mathrm{LD}}(n)$ grows when the field type is changing from zonal $(m=0)$ to sectorial $(m=n)$. In the meridional case the zonal terms contribute strongest and the sectorial terms weakest to the power spectrum of SV (except $y(n, m)$ which is zero when $m<1$ ).

As predicted by LOWES (1974) and demonstrated by a dipole model by ALLDREDGE (1984a) and by spherical harmonic models by NEVANLINNA (1987), the 
spectrum $Q_{\mathrm{LD}}(n)$ decreases as a function of $n$ much more slower than the energy density and its time derivative spectrum. In Fig. 2 we can see that the linear leastsquares fits of $Q_{\mathrm{LD}}(n)$ and $Q_{\mathrm{MD}}(n)$ are both decreasing when $n>2$. Their slope coefficients are -0.32 and -0.26 , respectively which are roughly half of the typical value $(-0.55)$ for $\dot{W}$. From the formula (7) for $Q_{\mathrm{LD}}(n)$ we can see that the factor $m^{2}$ is mainly responsible to the lower slope of the least-squares line for $Q_{\mathrm{LD}}(n)$ in Fig. 2. $\mathrm{m}^{2}$ is growing rapidly for high harmonics causing an increasing contribution to the spectrum $Q_{\mathrm{LD}}(n)$.

\section{Drift Velocities and Spatial Spectra}

Figure 1 shows the $Q_{\mathrm{LD}}(n)$ and $Q_{\mathrm{MD}}(n)$ curves for the IGRF 1980 supposing $u_{n}^{m}=v_{n}^{m}=0.25^{\circ} /$ year for all harmonics. It can be seen that $Q_{\mathrm{MD}}(n)$ is always greater than $Q_{\mathrm{LD}}(n)$ indicating that the gradients in spherical harmonic field are greater in the meridional direction than in the longitudinal one. Otherwise the shape of the two curves are similar but the dipole terms (axial+equatorial) are quite different. The difference is due to the drift of the axial dipole field which is by definition zero in the longitudinal case $(m=0)$ (see Eq. (7)) but non-zero in the meridional direction. Because the axial dipole term in the spatial spectrum of the field $(W)$ is roughly 10 times higher than its value expected from the linear trend determined by higher harmonics (see NEVANLINNA, 1987), the term $Q_{\mathrm{MD}}(1)$ will also differ clearly from the trend determined by the other harmonics. Note that even such a small MD drift velocity as $0.005^{\circ}$ / year in the axial dipole field would be enough to increase the dipole term $Q_{\mathrm{MD}}(1)$ as much as an equatorial drift of $0.15^{\circ} /$ year the $Q_{\mathrm{LD}}(1)$.

For determinations of $Q_{\mathrm{LD}}(n)$ the drift velocities $u_{n}^{m}$ are needed. We can use the formula given by WHITHAM (1958) which can be derived by the least-squares principle. The formula is (see also NAGATA, 1965; YUKUTAKE and TACHINAKA, 1968; JAMES, 1969):

$$
u_{n}^{m}=(1 / m)\left[\dot{g}_{n}^{m} h_{n}^{m}-h_{n}^{m} g_{n}^{m}\right] /\left[\left(g_{n}^{m}\right)^{2}+\left(h_{n}^{m}\right)^{2}\right], \quad m>0 .
$$

By putting this to (7) we get for $Q_{\mathrm{LD}}(n)$ :

$$
Q_{\mathrm{LD}}(n)=(n+1) \sum_{m=0}^{n}\left[\dot{g}_{n}^{m} h_{n}^{m}-h_{n}^{m} g_{n}^{m}\right]^{2} /\left[\left(g_{n}^{m}\right)^{2}+\left(h_{n}^{m}\right)^{2}\right]
$$

For Fig. 2 the velocities were calculated for 5 epochs (1965, 1970, 1975, 1980 and 1985) using spherical harmonic models published by IAGA DIVISION I WORKING GROUP 1 (1985). (The secular variation coefficients were calculated here using the 10 years differences except for the epoch 1985 for which the SV coefficients were given by the Working Group). Most of the harmonic constituents are drifting westward as is well known. For example in the IGRF 198024 from 36 pairs of the Gauss coefficients $(g, h)$ describe westward drifting field and their mean velocity is $0.31^{\circ} /$ year. The average eastward velocity was $0.24^{\circ}$ / year. The most rapid westward drifting terms are $(2,2)$, 


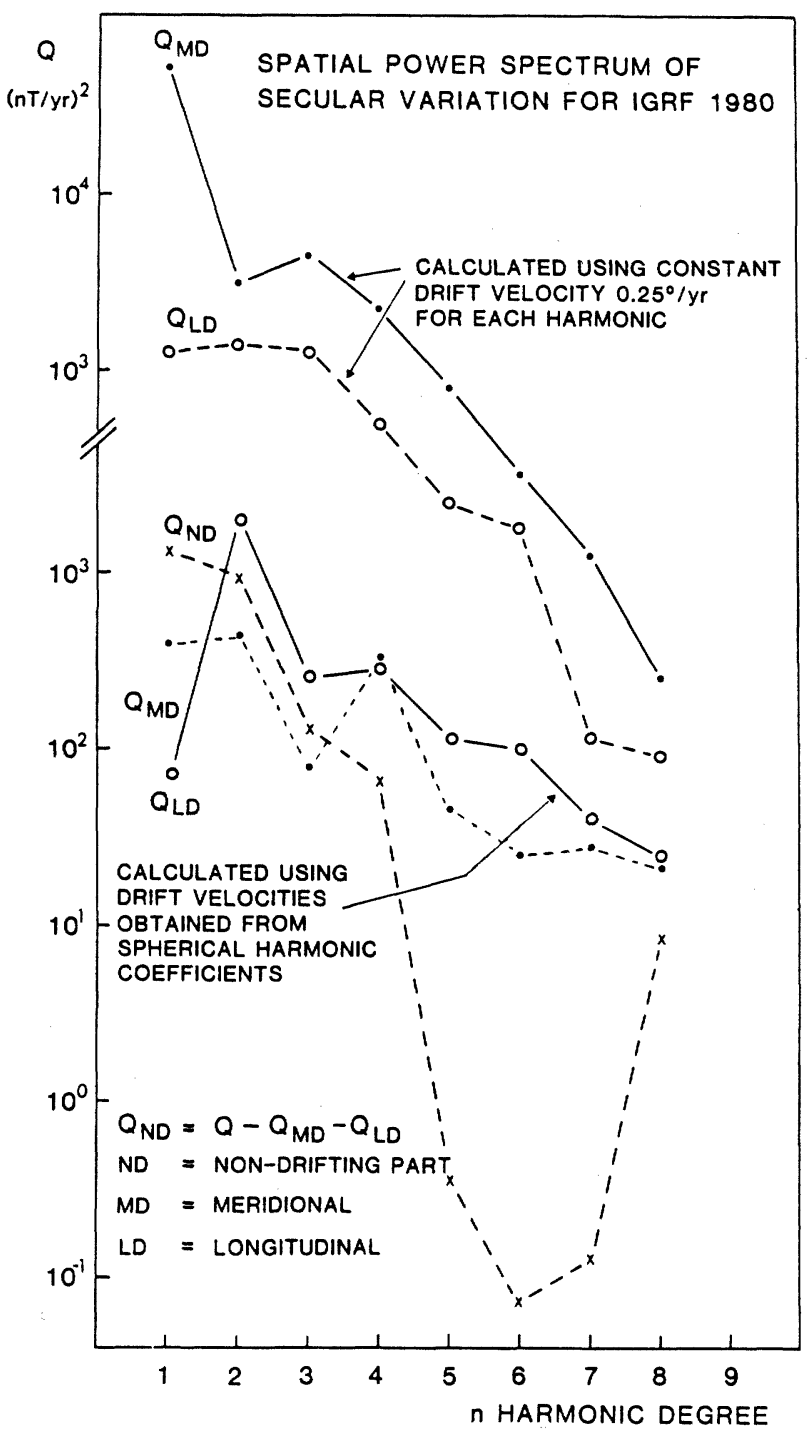

Fig. 1. Spatial power spectrum of the drifting ( $\mathrm{LD}=$ longitudinal direction; $\mathrm{MD}=$ meridional direction) secular variation as calculated for the IGRF 1980. The two uppermost curves have been calculated using a constant drift velocity of $0.25^{\circ}$ year. The three curves in the bottom show the LD and MD parts together with the non-drifting (ND) component. The LD and MD curves have been calculated using Eqs. (10) and (11). 


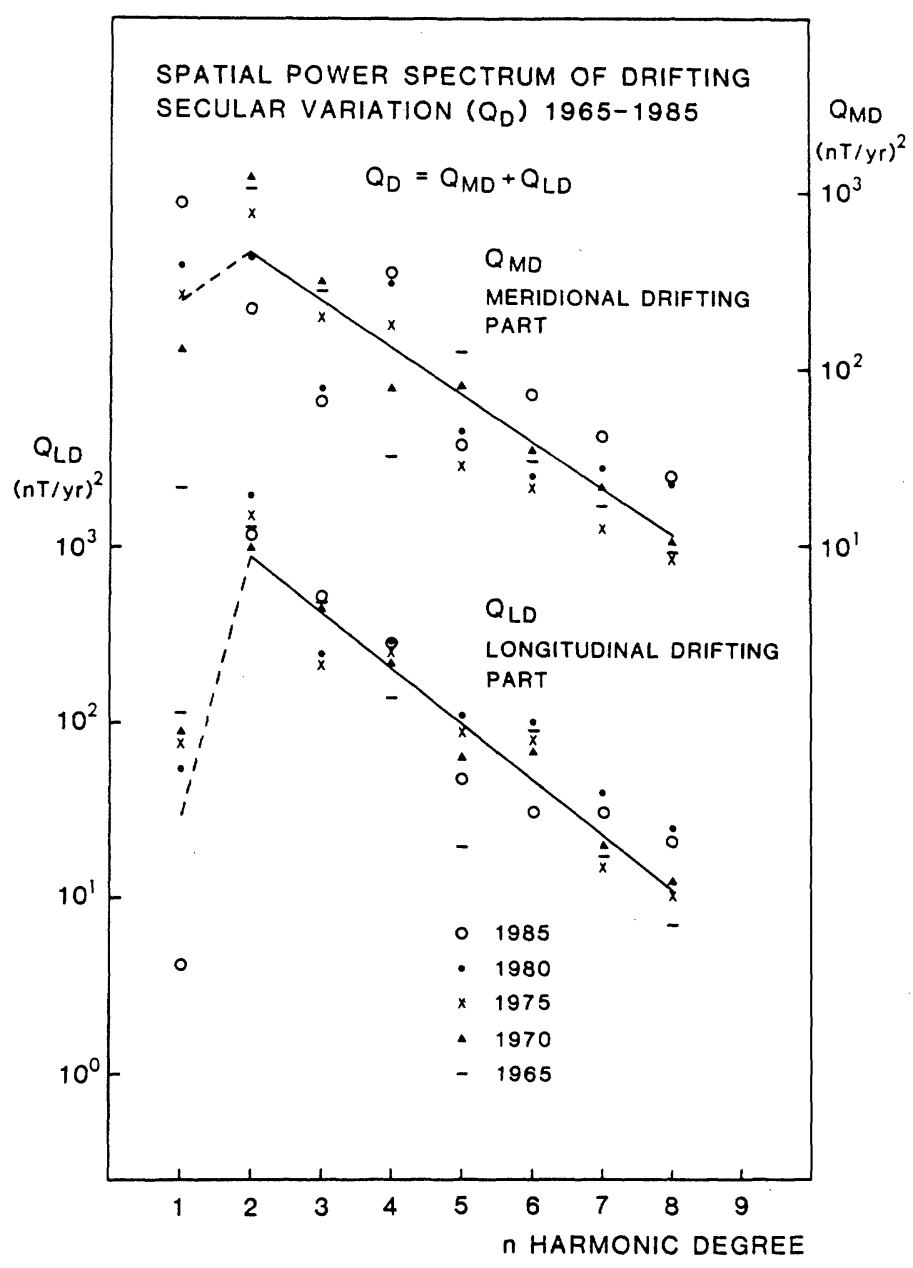

Fig. 2. Spatial power spectrum of drifting secular variation for the interval 1965-1985 as obtained from spherical harmonic coefficients published by IAGA DIVISION I WORKING GROUP 1 (1985). The solid lines are least-squares fit to the values $n>1$.

$(6,2)$ and $(7,6)$. Their drift velocities are $0.41,1.03$ and $1.76^{\circ} /$ year, respectively. The corresponding eastward drifting terms are $(8,8),(6,5)$ and $(4,1)$ with velocities 0.55 , 0.44 and $0.33^{\circ} /$ year, respectively. These velocities have been high during the last 20 years.

For the calculations of the MD part the formulas of the drift velocities are not necessarily needed because $Q_{\mathrm{MD}}(n)=Q_{\mathrm{D}}(n)-Q_{\mathrm{LD}}(n) . Q_{\mathrm{D}}(n)$ can be expressed in terms of $W, \dot{W}$ and $Q$ as shown by NEVANLINNA (1987). We thus get: 


$$
Q_{\mathrm{MD}}(n)=Q(n)-Q_{\mathrm{LD}}(n)-\dot{W}^{2}(n) / 4 W(n) .
$$

Using (11) and (8) we can calculate the numerical values for the drift velocities. The exact formulae of the velocity equations are quite complicated but for the zonal case we get the following simple formula:

$$
\left(v_{n}^{0}\right)^{2}=\left(\dot{g}_{n}^{0} / g_{n}^{0}\right)^{2}\left[n / 2+(n+1)^{2} /(2 n+1)\right]^{-1} .
$$

For the first 8 harmonics the mean MD velocity during 1965-1985 was $0.10^{\circ} /$ year. The axial dipole has been drifting slowest with a mean velocity of $0.007^{\circ} /$ year and the axial quadrupole most rapidly $0.29^{\circ} /$ year.

For non-zonal terms only a mean value of the MD velocities were calculated for each $n$. For the 20 years time interval studied here the averaged value for the 8 harmonics was $0.06^{\circ} /$ year.

Figure 1 shows the three parts $Q_{\mathrm{LD}}(n), Q_{\mathrm{MD}}(n)$ and $Q_{\mathrm{ND}}(n)$ of $Q(n)$ as calculated from the IGRF 1980. Because the MD drift velocities are in general almost one order of magnitude smaller than the corresponding LD ones, the MD part of the spectrum is smaller than the LD part except for the dipole and sedecimupole $(n=4)$ (for the names of higher harmonics, see WINCH, 1967a, b). The dipole term is unique because it is the only one in which the ND component dominates the drifting terms.

If the value of $Q(n)$ for each $n$ is normalized to 100 , the mean relative magnitudes (over $n=1, \ldots, 8$ ) of the LD, MD and ND parts for the IGRF 1980 are 51, 29 and 20, respectively. Thus the longitudinal part clearly dominates the secular variation spectrum. In 1965 these relative values were: 42, 35 and 23. This means that the longitudinal drifting part has increased slightly more than the meridional part has decreased.

In Fig. 2 there are shown the MD and LD parts of $Q(n)$ at five years interval since 1965. We can see that the values of the LD part shows much less scatter than the MD values from epoch to epoch. The MD component in the dipole field has increased especially much since 1965 . In 1965 its relative magnitude was only $2 \%$ (from the total dipole part of $Q$ ) but in 1985 it was $37 \%$. During the same time the LD part decreased from $21 \%$ down to $0.2 \%$.

Figure 3 shows the bulk spectrum, i.e. the sums $\Sigma_{n} Q_{\mathrm{LD}}(n), \Sigma_{n} Q_{\mathrm{MD}}(n)$ and $\Sigma_{n} Q_{\mathrm{ND}}(n)$ since 1955 . We can see that there was a minimum in the sum curve for the LD part around the year 1970 at which the so-called geomagnetic jerk was observed (see e.g. MALIN et al., 1983). In the MD part there was a maximum in 1970. The ND part has increased almost linearly since 1960 and no change in its curvature can be seen in 1970. The same parameters (for the years 1960-1980) as shown in Fig. 3 were also calculated using a continuous SV model by LANGEL et al. (1982). In their model the Gauss coefficients were represented by a truncated Taylor serie with first, second and third time derivatives. These curves showed the same long-term trend as found here, i.e. a broad minimum in $\Sigma Q_{\mathrm{LD}}$, maximum in $\Sigma Q_{\mathrm{MD}}$ around the year 1970 and a linear increase for $\Sigma Q_{\mathrm{ND}}$. These indicate that the jerk, if real, was connected with the drifting part of the SV field as also pointed out by e.g. COURTILLOT and LEMOUEL 


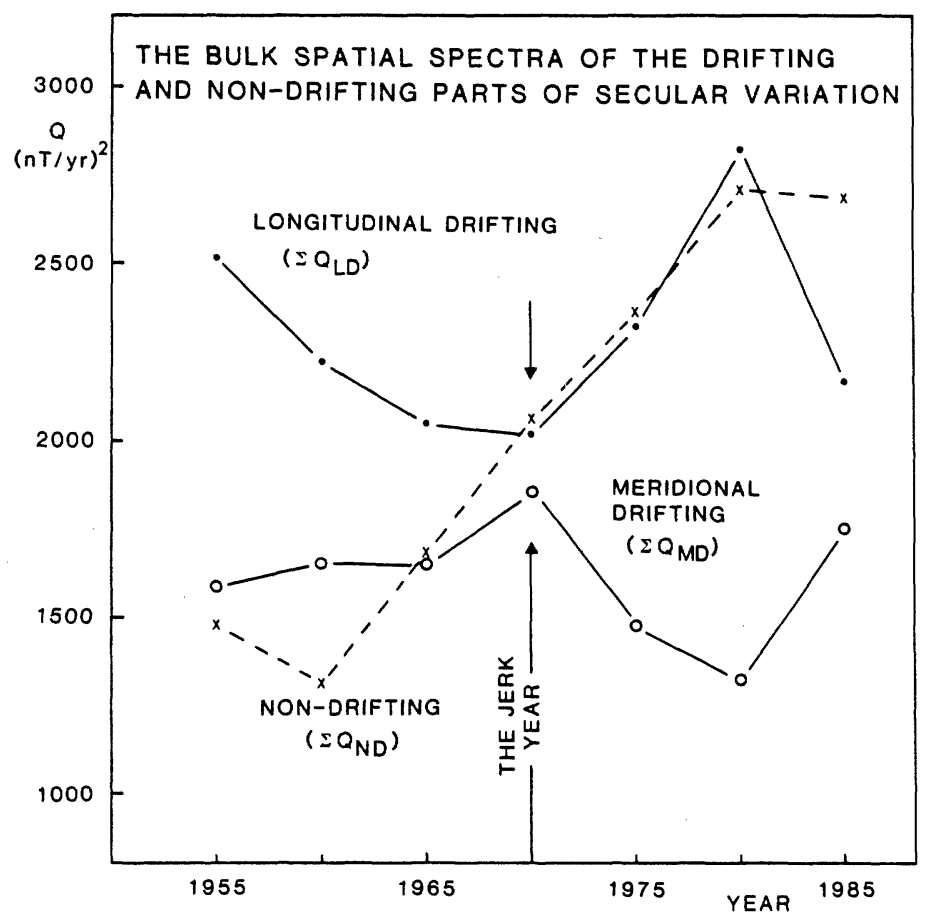

Fig. 3. The bulk spatial spectra defined as $\Sigma_{n} Q_{\mathrm{LD}}(n), \Sigma_{n} Q_{\mathrm{MD}}(n)$ and $\Sigma_{n} Q_{\mathrm{ND}}(n)$. Note the abrupt change in the curvature in the jerk year 1970. The anomalous behaviour of the points for 1985 is either real or caused by inaccuracies in the secular variation model for 1985 .

(1984) and NEVANLINNA (1984).

Note that the SV coefficients used in this study were determined as 10 years differences so we cannot draw conclusions on the crucial question in the jerk debate how rapid was the change around the jerk year 1970, did it happen in 1-2 years as claimed by e.g. MALIN et al. (1983).

The sum of the three sums defined above is exactly the same parameter $F(t)$ used by ALLDREDGE (1985). He did not find any change in that parameter which could be correlated with the jerk signal. However, as we can see in Fig. 3, the curves for the LD and MD parts are mutually anticorrelated and thus their changes compensate each other in the $F(t)$ curve used by Alldredge.

$\Sigma_{n} Q_{\mathrm{ND}}(n)$ exceeded $\Sigma_{n} Q_{\mathrm{MD}}(n)$ in 1965 and has been roughly equal to $\Sigma_{n} Q_{\mathrm{LD}}(n)$ since 1970 . Both $\Sigma_{n} Q_{\mathrm{LD}}(n)$ and $\Sigma_{n} Q_{\mathrm{ND}}(n)$ are now twice as large as $\Sigma_{n} Q_{\mathrm{MD}}(n)$.

As can be seen in Fig. 3, the year 1985 seems to be anomalous because the values of MD and LD curves differ considerably from the preceding ones. However, it is yet difficult to judge whether or not these latest changes are real or are they artificially caused by inaccuracies in the SV spherical harmonic model for 1985. On the other 
hand when using a recent SV model for 1987.5 (QUINN et al., 1986) the $\Sigma_{n} Q_{\mathrm{MD}}(n)$ part has been continued to increase very rapidly. This is mostly caused by the meridional drift of the dipole field as is predicted in Fig. 1.

The LD and MD velocities and the relative magnitudes of LD, MD and ND parts agree with the results by JAMES $(1969,1970)$ and WHITHAM $(1958)$ although their analyzing methods were not directly comparable with the present ones.

\section{Discussion and Conclusions}

The spatial spectrum of secular variation $Q(n)$ which is determined by the squares of the secular variation coefficients $g_{n}^{m}$ and $h_{n}^{m}$, can be divided into standing and drifting parts. The standing part of $Q$ describes secular variation due to intensity changes in the field and the drifting part represents secular variation caused by movements of the constant fieldline pattern. The drifting part can also be decomposed into two parts: the longitudinal and meridional drifting components. In the spatial spectrum of secular variation it is the longitudinal component which dominates. In the whole spectrum its relative part is typically $50 \%$ although in some harmonic constituents like in the recent secular variation of the dipole field it is not so pronounced. The mean meridional part is typically $30 \%$ and the non-drifting part $20 \%$ in the secular variation spectrum.

Typical longitudinal drift velocity is $0.3^{\circ} /$ year. In the meridional direction the velocities are in most harmonics less than $0.1^{\circ} /$ year. Among the harmonic constituents the quadrupole field has the most rapid drift in the meridional direction.

During the last 20 years the meridional drifting part of the secular variation spectrum has changed most. Especially the dipole and sedecimupole $(n=4)$ parts have changed much but the changes in adjacent harmonics are usually in the opposite direction so the bulk meridional spectrum does not show large differences from one epoch to the next.

There was a clear change in the curvature in the bulk spectrum of the drifting part but nothing in the non-drifting part around the jerk year 1970.

\section{REFERENCES}

ALLDREDGE, L. R., Harmonics required in main field and secular variation models, J. Geomag. Geoelectr., 36, 63-72, 1984a.

ALLDREDGE, L. R., Geomagnetic secular variation and varying dipoles in the core, J. Geomag. Geoelectr., 36, 621-633, 1984b.

Alldredge, L. R., More on alledged 1970 jerk, Phys. Earth Planet. Inter., 39, 255-264, 1985.

ALLDREDGE, L. R., Comments on "Remark on the secular change in the energy density spectrum of the geomagnetic field" by Joachim Meyer, J. Geomag. Geoelectr., 38, 171-172, 1986.

Chapman, S. and J. Bartels, Geomagnetism, Vol. II, Chp. XVII, Oxford at the Clarendon Press, London, 1940.

COURTILlot, V. and J.-L. LEMouel, On Backus's mantle filter theory and the 1969 geomagnetic impulse, J. R. Astron. Soc., 78, 619-625, 1984.

IAGA DIVISION I WORKING Group 1, International geomagnetic reference field revision 1985, J. Geomag. Geoelectr., 37, 1157-1163, 1985. 
James, R. W., Multipole analysis II. Geomagnetic secular variation, Aust. J. Phys., 22, 481-495, 1969.

JAMES, R. W., Decomposition of geomagnetic secular variation into drifting and non-drifting components, J. Geomag. Geoelectr., 22, 241-252, 1970.

LANGEl, R. A., R. H. Estes, and G. D. MEAD, Some new methods in geomagnetic field modeling applied to the 1960-1980 epoch, J. Geomag. Geoelectr., 34, 327-349, 1982.

LOWES, F. J., Spatial power spectrum of the main geomagnetic field, and extrapolation to the core, Geophys. J. R. Astron. Soc., 36, 717-730, 1974.

Malin, S. R. C., B. M. Hodder, and D. R. BARRAClOUGH, Geomagnetic secular variation-a jerk in 1970, in 75th Anniversary Volume of Ebro Observatory, edited by J. O. Cardus, pp. 239-256, Tarragona, Spain, 1983.

Meyer, J., Secular variation of magnetic mean energy density at the source layer depth, Phys. Earth Planet. Inter., 39, 288-292, 1985.

MEYER, J., Remark on the secular change in the energy density spectrum of the geomagnetic field, $J$. Geomag. Geoelectr., 38, 165-170, 1986.

Meyer, J., J.-H. Hufen, M. Siebert, and A. HAhn, Investigations of the internal geomagnetic field by means of a global model of the Earth's crust, J. Geophys., 52, 71-84, 1983.

NAGATA, T., Main characteristics of recent geomagnetic secular variation, J. Geomag. Geoelectr., 17, 263-276, 1965.

Nevanlinna, H., Some characteristics of the horizontal field variations around the geomagnetic jerk of 1970, J. Geophys., 55, 37-40, 1984.

Nevanlinna, H., Notes on global mean-square values of the geomagnetic field and secular variation, $J$. Geomag. Geoelectr., 39, 165-174, 1987.

Ostrowski, J. A., Spectra of the international geomagnetic reference field and its secular variation, $J$. Geomag. Geoelectr., 34, 417-422, 1982.

Quinn, J. M., D. J. KERRIDGE, and D. R. BARRAClough, World magnetic charts for 1985-spherical harmonic models of the geomagnetic field and its secular variation, Geophys. J. R. Astron. Soc., 87, 1143-1158, 1986.

Whiтнам, K., The relationship between the secular change and the nondipole fields, Can. J. Phys., 36, 1372-1396, 1958.

Winch, D. E., The fourth order geomagnetic multipole: The sedecimupole, Pure Appl. Geophys., 67, 112-122, 1967a.

Winch, D. E., The fifth order geomagnetic multipole: The duotrigintupole, Pure Appl. Geophys., 68, 90-102, 1967b.

Yukutake, T. and H. TachinaKa, The non-dipole part of the Earth's magnetic field, Bull. Earthq. Res. Inst. Tokyo Univ., 46, 1027-1074, 1968. 